Revista de

CIENCIAS AMBIENTALES

Tropical Journal of Environmental Sciences

\title{
EXPERIENCIAS
}

\section{La experiencia de Caminos de Osa: una iniciativa de turismo sostenible en Costa Rica}

The Experience of Caminos de Osa: an Initiative of Sustainable Tourism in Costa Rica

\section{Erick Vargas Campos ${ }^{\mathrm{a}}$, Emily Arnold ${ }^{\mathrm{b}}$, Diego García Godínez ${ }^{\mathfrak{c}}$}

a Presidente de Sostenible por Naturaleza; excoordinador nacional de la Iniciativa Osa y Golfito, Costa Rica erick927@icloud.com

b Directora asociada, Iniciativa Osa y Golfito, Costa Rica, ebarnold@stanford.edu

c Presidente, Asociación Caminos de Osa, Costa Rica, dgarciagodinez@hotmail.com

Director y Editor:

Dr. Sergio A. Molina-Murillo

Consejo Editorial:

Dra. Mónica Araya, Costa Rica Limpia, Costa Rica Dr. Gerardo Ávalos-Rodríguez. SFS y UCR, USA y Costa Rica Dr. Olman Murillo Gamboa, ITCR, Costa Rica

Dr. Luko Hilje, CATIE, Costa Rica

Dr. Arturo Sánchez Azofeifa. Universidad de Alberta-Canadá

Asistente:

Joseline Jimenez Brenes

Editorial:

Editorial de la Universidad Nacional de Costa Rica (EUNA)
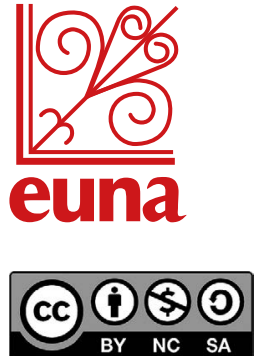

Los artículos publicados se distribuyen bajo una licencia Creative Commons Reconocimiento al autor-No comercial-Compartir igual 4.0 Internacional (CC BY NCSA 4.0 Internacional) basada en una obra en http://www.revistas.una.ac.cr/ambientales, lo que implica la posibilidad de que los lectores puedan de forma gratuita descargar, almacenar, copiar y distribuir la versión final aprobada y publicada (post print) del artículo, siempre y cuando se realice sin fines comerciales, no se generen obras derivadas y se mencione la fuente y autoría de la obra. 


\title{
EXPERIENCIAS
}

\section{La experiencia de Caminos de Osa: una iniciativa de turismo sostenible en Costa Rica}

\author{
The Experience of Caminos de Osa: an Initiative of Sustainable Tourism in Costa Rica
}

\author{
Erick Vargas Campos ${ }^{\mathrm{a}}$, Emily Arnold ${ }^{\mathrm{b}}$, Diego García Godínez
}

\begin{abstract}
Resumen
Caminos de Osa es un esfuerzo que busca consolidar un destino de turismo sostenible, a partir de emprendimientos locales, tanto familiares como personales, para el desarrollo local integral y sostenible de la península de Osa. Este ha venido tomando forma desde el 2011 con el establecimiento de la Iniciativa Osa y Golfito (INOGO) del Stanford Woods Institute for the Environment (Stanford University) y despegó en el 2014 gracias a la alianza con otros 3 socios: el Área de Conservación Osa (ACOSA), la Fundación CRUSA y la empresa Reinventing Business for All (RBA). Cuenta, además, con apoyo de muchos actores públicos, privados, no gubernamentales y académicos. Caminos de Osa busca promover el emprendedurismo local, desarrollar una oferta de productos y servicios turísticos de calidad y fortalecer la cadena de comercialización. Diversos emprendedores participantes fundaron la Asociación Caminos de Osa (ACO) como modelo de gobernanza, para dar continuidad y sostenibilidad al proceso en el largo plazo.
\end{abstract}

Palabras clave: alianzas; conservación; desarrollo local; emprendimiento; turismo rural

\begin{abstract}
Caminos de Osa is an effort to consolidate a sustainable tourism destination from local individual and family ventures, for the integral and sustainable local development of the Osa Peninsula. It took shape since 2011 with the establishment of the Osa and Golfito Initiative (INOGO) by the Stanford Woods Institute for the Environment (Stanford University) and took off in 2014 thanks to an alliance with three other partners: the Osa Conservation Area (ACOSA), CRUSA Foundation, and Reinventing Business for All (RBA). It also has the support of many other public, private, nongovernmental and academic actors. Caminos de Osa seeks to promote local entrepreneurship, develop quality tourism products and services, and strengthen the commercialization chain. Several entrepreneurs participating in the project founded the Caminos de Osa Association (ACO) as a model of governance to provide long term continuity and sustainability.
\end{abstract}

Keywords: alliances; conservation; entrepreneurship; local development; rural tourism

\footnotetext{
a Presidente de Sostenible por Naturaleza; excoordinador nacional de la Iniciativa Osa y Golfito, Costa Rica erick927@icloud.com

a Presidente de Sostenible por Naturaleza; excoordinador nacional de la Iniciativa Osa y Golfito, Costa Rica erick927@icloud.com
b Directora asociada, Iniciativa Osa y Golfito, Costa Rica, ebarnold@stanford.edu

c Directora asociada, Iniciativa Osa y Golfito, Costa Rica, ebarnold@stanford.edu
}

Creative Commons (Reconocimiento al autor-No comercial-Compartir igual 4.0 Internacional (CC BY NC SA 4.0 Internacional) 


\section{Introducción}

\subsection{El contexto de la península de Osa}

\subsubsection{Ubicación y recursos naturales}

La península de Osa se ubica en el Pacífico Sur de Costa Rica, en la Región Brunca, y es compartida por los cantones de Osa y Golfito. Según la regionalización del Ministerio de Ambiente, la península se ubica en el Área de Conservación Osa, ACOSA, del Sistema Nacional de Áreas de Conservación, SINAC.

El área sobresale por sus amplias reservas de capital natural y los servicios ecosistémicos que proveen. La península de Osa alberga $2.5 \%$ de la biodiversidad mundial y más del $50 \%$ de la biodiversidad de Costa Rica (Toft y Larsen, 2009, citados por Dirzo et al., 2014) siendo, con relación al área que abarca, una de las regiones con mayor densidad de diversidad biológica en el mundo (Barquero et al., 2012, citados por Dirzo et al., 2014). Incluye bosques tropicales lluviosos con extensión suficiente para mantener poblaciones viables de especies representativas de la flora y fauna mesoamericanas, por lo que desde los años 1970 ha sido reconocida como un área prioritaria para la conservación (Cornejo et al., 2012, citados por Dirzo et al., 2014).

\subsubsection{Contexto económico y social}

La zona se destaca por sus contradicciones entre crecimiento y conservación. Desde finales de 1930, el Estado ha promovido grandes monocultivos, primero banano y actualmente palma africana. El Gobierno también comprometió amplias extensiones del territorio a la conservación, creando en 1975 el Parque Nacional Corcovado (42 570 hectáreas terrestres y 5375 marinas) y en 1979 la Reserva Forestal Golfo Dulce (casi 70000 hectáreas). Esta dualidad explica en gran medida la dinámica social, económica y ambiental de la región, ya que la manera en que se ha implementado el modelo de conservación dificulta generar prosperidad en las comunidades.

La principal actividad es la agricultura, donde la palma africana se viene expandiendo en detrimento del arroz y el ganado (Román y Angulo, 2013). La población local se ha visto en la necesidad de buscar otras opciones como el cultivo del cacao y la explotación turística rural, relacionadas con sus actividades productivas. El turismo de naturaleza también ha venido creciendo, ligado a Corcovado y otros atractivos.

En un contexto de gran riqueza biológica con producción agropecuaria y turismo, las poblaciones enfrentan desafíos y oportunidades para lograr mayor bienestar. En salud, el incremento en el embarazo adolescente, la drogadicción y el alcoholismo en jóvenes, afectan severamente el tejido social de las comunidades (Gaffikin, 2013). En educación, el abandono de los estudios aumenta dramáticamente a medida que los alumnos avanzan en el sistema, ya que una instrucción poco diversificada y la escasez de empleo hacen que muchos no vean la necesidad de estudiar (Menke y Carnoy, 2013). En general, los líderes de las comunidades de la península y otros sectores de ACOSA identifican a la naturaleza como la principal fortaleza de la región, seguida de

\begin{tabular}{|c|c|c|}
\hline 218 & $\begin{array}{l}\text { Creative Commons (Reconocimiento al autor-No comercial-Compartir igual } 4.0 \text { Internacional } \\
\text { (CC BY NC SA } 4.0 \text { Internacional) }\end{array}$ & $\begin{array}{l}\text { Revista de } \\
\text { CIENCIAS AMBIENTALES } \\
\text { Tropical Journal of Environmental Sciences }\end{array}$ \\
\hline
\end{tabular}


la identidad cultural (Hunt, Menke y Durham, 2013). También, tienen una percepción favorable del turismo como actividad altamente sostenible en lo ambiental y lo económico.

\subsubsection{El turismo en la península de Osa}

Muchos turistas escogen la península de Osa porque cuenta con un 80 \% de su territorio bajo conservación y alberga el $2.5 \%$ de la biodiversidad del planeta. Sin embargo, el turismo se concentra en pocos oferentes de servicios, principalmente en Corcovado, con un aumento en la visitación que provoca su saturación.

Las comunidades del centro de la península son sitios de paso, quedando rezagadas en sus ofertas turísticas. La actividad sufre además de problemas que frenan la participación de los actores locales en los beneficios, entre estos: la falta de consolidación de la región como destino integral con una imagen definida, débil autoestima entre los pobladores, falta de una clara identidad cultural, insuficiente calidad de los productos y servicios turísticos y débiles canales para su comercialización.

También hay que agregar la carencia de un acompañamiento técnico y estratégico más allá de eventos puntuales de capacitación y la falta de una plataforma de autogestión regional. Para mejorar las perspectivas del turismo es necesario desarrollar los atractivos, mejorar la calidad de los productos y servicios locales, armar una oferta de experiencias atractivas para el mercado, fortalecer los encadenamientos comerciales y generar oportunidades para las familias que conviven con la rica biodiversidad de la región. Es en este marco, que una alianza de socios estratégicos promueve la consolidación del destino Caminos de Osa, como una oportunidad para el desarrollo local integral y sostenible.

\subsubsection{El giro de conservación a producción local sostenible}

En la península de Osa conviven varias ONG que fueron creadas con fines de conservación. Si bien algunas vienen apoyando actividades productivas sostenibles desde finales de los 80 , es cada vez más evidente el cambio que se ha venido dando en casi todas ellas desde mediados de la última década, de un trabajo enfocado en conservación a otro que apunta a la creación de capacidades, apoyo a emprendimientos locales y mecanismos de microcrédito para grupos de base. La premisa es que un mayor bienestar con sostenibilidad de los pobladores locales contribuye en el largo plazo a una conservación más efectiva de la biodiversidad y de las áreas silvestres protegidas.

La rica experiencia sirve de base a los esfuerzos realizados por Caminos de Osa a partir del 2014. Un antecedente importante fue el proyecto de la Fundación Neotrópica Liderazgo Juvenil Empresarial (2002-2004) apoyado por la Fundación AVINA. Su propósito fue promover en la península de Osa la creación de pequeñas y medianas empresas con criterios de calidad ambiental, rentabilidad económica y beneficio social. Se brindó capacitación a hombres y mujeres jóvenes en temas empresariales y se dio apoyo en el diseño de sus planes de negocios. De las 20 iniciativas participantes, 13 se enfocaron en actividades asociadas al ecoturismo.

Desde el sector gubernamental también ha habido algunos aportes. Uno de ellos es el-programa Avancemos Mujeres del Instituto Nacional de las Mujeres (INAMU) y el Instituto Mixto 
de Ayuda Social (IMAS), que en el 2012 apoyó en Rancho Quemado a 16 emprendimientos de mujeres. Otra entidad pública que ha ofrecido apoyo en la zona es el INA, con capacitaciones en manipulación de alimentos, cocina para microempresarios y administración.

Desde junio del 2014 el Estado inició un interesante esfuerzo por atender los desafíos de la conservación en la península de Osa en general y del Parque Nacional Corcovado en particular. Este nació por iniciativa de la Presidencia de la República con el nombre genérico de Hoja de Ruta y luego pasó a llamarse Pongámole: Es tiempo de trabajar por la península de Osa. Esta experiencia abarca un periodo que va del 2014 hasta el 2018, cuya característica ha sido contar con una agenda de trabajo para la búsqueda de soluciones a la conservación de la biodiversidad en la península de Osa desde el Gobierno, por medio de la coordinación interinstitucional (MINAE, INDER, IMAS, CCSS, MIVAH, DINADECO, otras), el fortalecimiento de la presencia de varias entidades públicas y la alianza con iniciativas locales como Caminos de Osa y las lideradas por la Asociación Conservacionista de Dos Brazos de Río Tigre (ACODOBRARTI) y la Asociación de desarrollo Integral Corcovado Carate (ADICORCO), que trabajan directamente con públicos de interés, como personas oreras y exoreras.

\section{Una Alianza para el turismo sostenible en la península de Osa}

\subsection{La Iniciativa Osa y Golfito (INOGO)}

En el 2010 algunos distinguidos ciudadanos de Costa Rica y Estados Unidos interesados en la península de Osa decidieron ponerse en acción y trabajar con otras personas de ambos países en la búsqueda de recursos. Además, se dieron a la tarea de identificar una universidad de alto nivel, con capacidad para proponer opciones viables para el desarrollo sostenible de la región. Como resultado, lograron el compromiso de un donante y despertaron el interés del Stanford Woods Institute for The Environment, Universidad de Stanford (California). Allí se integró a la iniciativa el Profesor William H. Durham, antropólogo, quien a su vez invitó al profesor Rodolfo Dirzo, biólogo, ambos con amplia experiencia en Costa Rica y en Osa.

Desde un inicio, la intención fue construir sobre los esfuerzos previos en la región y trabajar con los líderes locales para definir con ellos el futuro que deseaban para sus familias y comunidades, así como identificar acciones tendientes a avanzar en la dirección deseada. Este esfuerzo se denominó Iniciativa Osa y Golfito, INOGO, en referencia a los dos cantones que ocupan la península de Osa.

Entre el 2011 y el 2014 INOGO desarrolló una primera fase de diálogo con los actores locales, para analizar las necesidades y oportunidades. Los resultados mostraron que, en general, a pesar de la presencia de numerosas ONG y organizaciones comunales, estas no trabajaban juntas ni se comunicaban unas con otras. También se detectó la necesidad de fortalecer el liderazgo con un enfoque en el desarrollo del capital social (Hunt, Menke y Durham, 2013), que es el que emerge de la interacción entre los actores y permite el acceso a los agentes de poder (representantes de ONG, funcionarios públicos, políticos, y otros). 
En 2014 INOGO se integró a una alianza estratégica con otros tres actores para fomentar el desarrollo de los líderes en la región por medio del proyecto Caminos de Osa. Paralelamente, viene desarrollando el Programa de Liderazgo Ambiental e Idioma de Stanford (SELAL) bajo la conducción del profesor Durham, que busca promover la conciencia y acción ambiental y facilitarles a los jóvenes el acceso a empleos en turismo local. INOGO desarrolla además el Laboratorio Experimental Palma Sostenible y Diversificada, (LAPA), dirigido por el profesor Dirzo, que explora las ventajas de la diversificación biológica de las plantaciones de palma aceitera.

\subsection{Diferentes socios, un mismo objetivo}

Al comenzar el 2014, cuatro socios se unieron para trabajar con el fin común de consolidar un modelo de desarrollo sostenible en la península de Osa, que mejore las condiciones de vida de la población, sin comprometer la integridad de los ecosistemas naturales que rodean a las comunidades. Estos socios son:

- Área de Conservación Osa (ACOSA): es el ente responsable de una de las 11 regiones administrativas de conservación en las que se divide el país y que componen el Sistema Nacional de Áreas de Conservación, SINAC. Su responsabilidad es la administración y preservación de la biodiversidad y de los recursos naturales en general. Aporta a Caminos de Osa su experiencia en la conservación de la biodiversidad y de las áreas protegidas.

- Fundación Costa Rica-Estados Unidos para la Cooperación (CRUSA): agencia de cooperación, que es una entidad privada costarricense y sin fines de lucro, orientada al apoyo de proyectos y la gestión de iniciativas de largo alcance, alianzas y redes de cooperación. CRUSA contribuye con financiamiento a Caminos de Osa.

- Universidad de Stanford y su Iniciativa Osa y Golfito (INOGO): es un actor académico que suma acompañamiento, conocimientos y fondos de contrapartida.

- Reinventing Business for All (RBA): es una empresa consultora, que impulsa la competitividad de organizaciones a través del diseño e implementación de modelos de negocio innovadores, creando valor a las empresas y a sus públicos de interés.

La Alianza, denominada Caminos de Liderazgo, se sustentó en la convicción conjunta de que la conservación futura de la península de Osa y de su biodiversidad depende de la calidad de vida de su gente y de la consolidación de un modelo regional de economía verde.

INOGO había aprendido de su fase I que la clave del desarrollo sostenible de la región estaba en las mismas comunidades locales, con sus organizaciones, líderes y empresas. Paralelamente, CRUSA había llegado a la misma conclusión y, de la mano con RBA, estaba diseñando un proyecto en esa línea denominado Caminos de Osa, enfocado en liderazgo y acceso a mercados. RBA sugirió el fortalecimiento de líderes y su integración en una organización de gestión del destino como figura de gobernanza. Esto llevó a las tres organizaciones a tomar la decisión de trabajar en forma conjunta. A ellas se sumó ACOSA, dada su importancia estratégica como un actor institucional principal con presencia en la región.

(1) 


\section{El Proyecto Caminos de Osa}

\subsection{Caminos de Osa: ¿en qué consiste?}

Los socios de la alianza se propusieron crear el destino Caminos de Osa, a través de un modelo de desarrollo turístico sostenible en la península de Osa, con una oferta de productos y servicios en manos de las familias locales, que respondiera al interés del turista de disfrutar de naturaleza y cultura, mejorando el bienestar y calidad de vida de los pobladores. Se propuso incluir emprendedores con servicios de hospedaje, alimentación, artesanías, excursiones, transporte guiado y otros. El proyecto se organizó en torno a tres componentes de fortalecimiento de capacidades individuales y locales:

1. Desarrollo social y empoderamiento comunitario: este componente busca promover un cambio interior en las personas y en sus vidas a partir de que el éxito viene del corazón, y no solo de recibir información o desarrollar habilidades. Se enfoca en el desarrollo de la autoestima y el liderazgo, por medio de juegos y dinámicas donde los participantes generan conocimiento acerca de sus propias vivencias. También busca hacer emerger el sentido de pertenencia e identidad local en el contexto de la península de Osa por medio del arte y la cultura, valorando y revitalizando la vida rústica rural.

2. Desarrollo del servicio y la infraestructura turística: el punto de partida es el entusiasmo y trabajo tenaz de pequeños emprendedores y sus familias, que han decidido desarrollar sus propios negocios. Este bloque se enfoca en mejorar la calidad de la oferta de los emprendimientos con acompañamiento y desarrollo de capacidades, de manera que puedan competir en el largo plazo. Busca consolidar la cadena de valor del turismo con encadenamientos entre los emprendedores, permitiendo ofrecer mayor diversidad. También se incluye un trabajo en alianza con destacadas turoperadoras nacionales.

3. Conceptualización del destino: se concentra en dar forma a la noción Caminos de Osa como destino turístico. En un proceso participativo, en el que emprendedores definen su visión del desarrollo turístico para la región, así como la identidad del destino y la experiencia que se quiere compartir con el turista. Incluye la creación de una red de senderos que conectan atractivos naturales y culturales en la península de Osa. 


\section{Reseña breve de Caminos de Osa}

Situación inicial: se busca que el derrame vaya a las comunidades en el interior de la península, ya que $80 \%$ de ingresos generados por turismo permanece en las aerolíneas y en hoteles cercanos a Corcovado o en la costa.

Objetivo socioeconómico de Caminos de Osa: promover el desarrollo integral de las comunidades, por medio de un turismo que combine naturaleza y cultura, encadenándolos a lo largo de tres rutas de peregrinaje. Juega un papel importante la Asociación Caminos de Osa, ACO, que busca ser una voz para las necesidades de la región, un canal legítimo para incidencia política y un comercializador del destino.

Objetivo ambiental de Caminos de Osa: contribuir con la conservación de las áreas protegidas de ACOSA, así como de sus servicios ecosistémicos.

Beneficiarios: 40 emprendimientos turísticos, cerca de 150 miembros de las familias, 5 turoperadoras locales, 50 emprendimientos locales no turísticos que sean complementarios, asociaciones de desarrollo integral, guías, turistas, otros.

Abordaje participativo: es un proceso construido desde la comunidad, que busca que esta sea la protagonista y la que asuma el liderazgo a través de la ACO. Se enfatiza en el desarrollo personal, el empoderamiento y la autoestima.

Coordinación interinstitucional: se trabaja de la mano con la institucionalidad del Estado (Presidencia, MINAE, MEIC, ICT, IMAS, INAMU, Consorcio de Turismo, Consejo de Competitividad de la Región Brunca).

\subsection{La primera generación de emprendedores (2014-2015)}

El proyecto apuesta a la capacitación de los participantes, como un medio indispensable para el desarrollo de sus conocimientos, habilidades y destrezas, así como a su empoderamiento y autoestima. Entre agosto del 2014 y mayo del 2015 se desarrolló el primer programa de capacitación, facilitado por RBA. Los participantes de la primera generación de emprendedores se identificaron a partir de personas de la región que ya eran conocidas por los miembros de la Alianza y que cumplían con dos criterios: 1) que el candidato tuviera liderazgo dentro de su comunidad y 2) que contaran con al menos una idea de emprendimiento. Se buscaba trabajar con líderes que incidieran en el desarrollo del destino. Los participantes seleccionados representaban comunidades como Sierpe, El Progreso, Drake, Rancho Quemado, La Tarde, Alto Laguna, La Palma y Playa Blanca.

Aunque la intención era armar un grupo homogéneo en experiencia y conocimientos, en la práctica se incluyeron participantes que estaban avanzados en sus emprendimientos y otros que apenas iniciaban, por lo que fue necesario atender necesidades e inquietudes de participantes de diversos niveles. De 30 participantes que iniciaron, 23 concluyeron con éxito (Figura 1). 


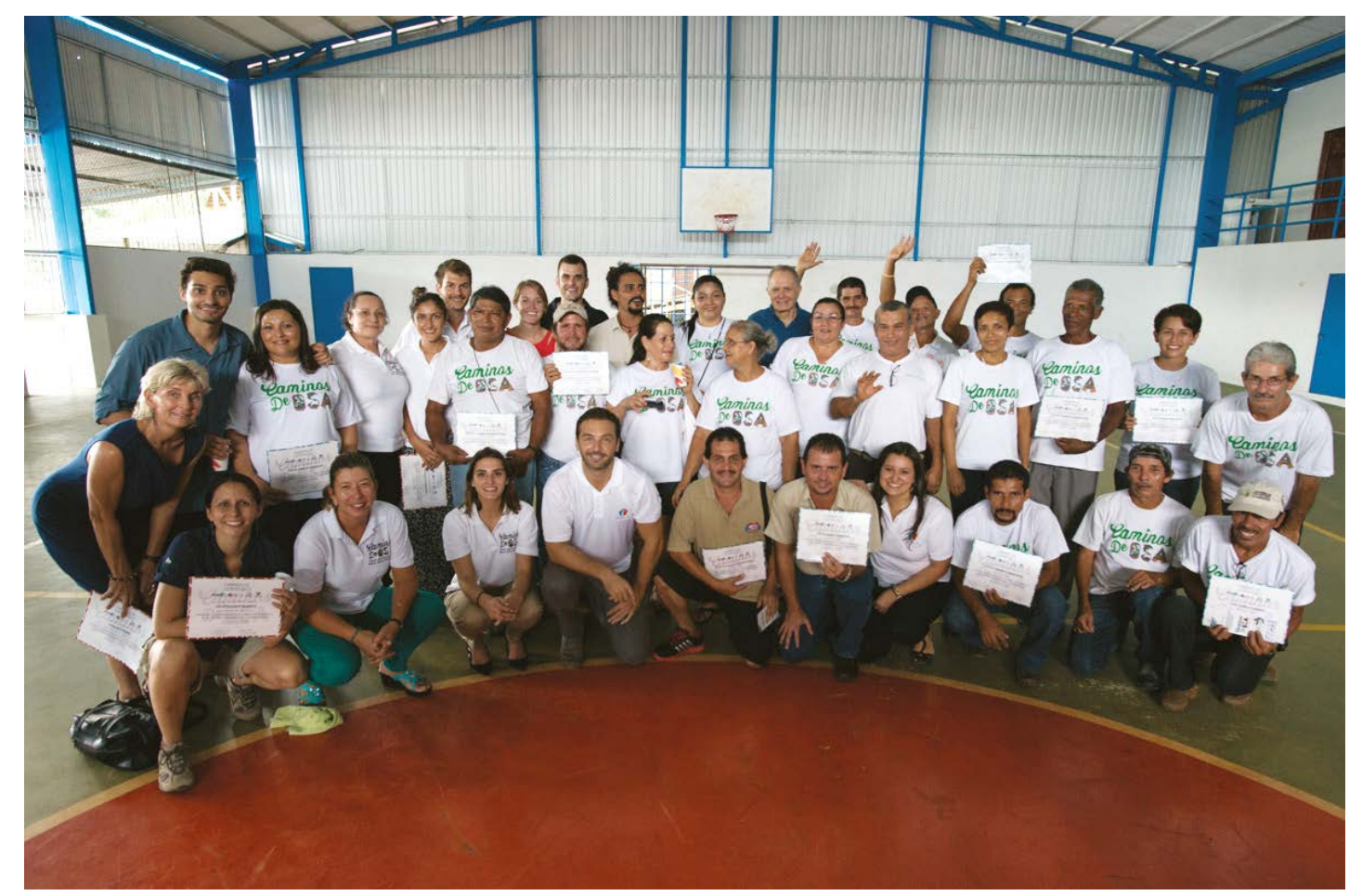

Figura 1. Graduación de la primera generación de participantes locales en Caminos de Osa. Fotografía: Fabiola Kano

\subsection{La segunda generación (2015-2016)}

La identificación y selección de candidatos para la segunda generación de Caminos de Osa se centró en incluir emprendimientos que diversificaran la oferta de atractivos y la variedad de excursiones por ofrecer. También se buscó incorporar a participantes de comunidades que no estuvieron representadas en la primera generación o que vinieran a llenar vacíos en los caminos. En esta ocasión, la ACO fue relevante en la convocatoria y selección de los participantes. Las recomendaciones de posibles candidatos vinieron de los mismos participantes de la primera generación, sus mentores y algunos aliados del proyecto. Entre los seleccionados hubo personas provenientes de Dos Brazos de Río Tigre, El Ñeque, Miramar y Estero Guerra, con servicios turísticos como alimentación, hospedaje y transporte, que venían a fortalecer las rutas.

Este segundo grupo de capacitación inició con 13 mujeres y 4 hombres. Un factor que podría explicar el predominio de mujeres es que para esta segunda generación las visitas de casa en casa fueron realizadas por dos mujeres miembros del equipo de trabajo. Esto pudo haber despertado el interés y la motivación de las esposas. También, al ser el turismo una nueva opción de ingresos familiares, es la mujer la que tiene mayor flexibilidad para explorar esta oportunidad, mientras que el hombre debía continuar con su trabajo para llevar el pan a la casa.

\begin{tabular}{|c|c|c|}
\hline 224 & $\begin{array}{l}\text { Creative Commons (Reconocimiento al autor-No comercial-Compartir igual } 4.0 \text { Internacional } \\
\text { (CC BY NC SA } 4.0 \text { Internacional) }\end{array}$ & $\begin{array}{l}\text { Revista de } \\
\text { CIENCIAS AMBIENTALES } \\
\text { Tropical Journal of Environmental Sciences }\end{array}$ \\
\hline
\end{tabular}




\section{Desarrollo social y empoderamiento comunitario}

\subsection{Empoderamiento personal y liderazgo}

En la península de Osa muchos pobladores enfrentan barreras psicológicas, que les dificultan el aprovechamiento de sus propios recursos personales para proponerse metas, trabajar por ellas y salir adelante. En este contexto, se busca desarrollar la autoestima y el empoderamiento en las personas, a través de un conjunto de talleres prácticos.

Con los integrantes de cada generación se realizó un diagnóstico de línea base para conocer sus habilidades de liderazgo, organización y comunicación, así como su nivel de motivación y el concepto de éxito que manejaban. Esto permitió identificar dificultades en su autoestima, en la percepción de sí mismos y de sus negocios.

A partir de allí, la capacitación desarrolló varios talleres: 1) Plan de vida, 2) Empoderamiento para el liderazgo, 3) Comunicación asertiva, 4) Disciplina y manejo del tiempo, 5) Trabajo en equipo, 6) Resolución de conflictos, 7) Empoderamiento para la equidad de género, y 8) Inteligencia emocional. Estos se abordaron desde la psicología constructivista, que permite a los participantes desarrollar el conocimiento a partir de sus propias vivencias.

Cada taller tuvo un manual a la medida del participante, con información concreta y visual, poco texto y fotografías de los mismos participantes para reforzar que ellos eran los protagonistas. También se ofrecieron ejercicios vivenciales que los invitaban a mirar hacia adentro, sopesar sus emociones y extraer lecciones aprendidas que quedaran gravadas en sus recuerdos y en sus vidas. Se les motivó además a expresarse con confianza, proponer ejemplos propios y compartir experiencias personales.

Estas capacitaciones fueron fundamentales para Caminos de Osa, ya que motivó a los participantes de ambas generaciones a irse empoderando rápidamente. Ellos comenzaron a creer y a verse a sí mismos en otro nivel como personas, líderes comunales y empresarios serios. También reconocieron sus propios estilos de comunicación y trabajaron en adoptar un lenguaje y una presentación personal acordes con sus roles de líderes y empresarios. Además, evolucionaron hacia un mayor aprecio de sus propias empresas.

El nivel educativo de las personas no fue un obstáculo para lograr el cambio esperado. Algunos participantes con baja escolaridad se esforzaron con un gran compromiso de aprender y concretaron muy rápidamente sus manuales de venta, con el apoyo de sus respectivos mentores. Por otro lado, varios participantes que ya contaban con una amplia capacitación en temas de turismo y administración reconocieron que aún tenían pendiente la tarea de empoderarse.

En síntesis, el empoderamiento personal y liderazgo en el contexto de Caminos de Osa significa:

- Sentirse orgulloso de tener un emprendimiento o negocio.

- Proyectar autoconfianza para posicionar y vender su servicio o producto.

- Verse a sí mismo no como una víctima, sino como un actor del cambio.

- Interiorizar la importancia del trabajo en equipo, en función del bien común.

- Empoderar a Caminos de Osa, al tomar en sus propias manos las riendas del proceso. 


\subsection{Medio ambiente}

Caminos de Osa parte de que la conservación pasa por nuevas opciones de desarrollo sostenible, por ende, el turismo asociado a la naturaleza es una de ellas. El tema ambiental busca que los emprendimientos locales se consoliden como negocios verdes con conciencia pro ambiente, aplicando en su operación criterios amigables con él. Estos incluyen conocimiento de la naturaleza, gestión del recurso hídrico, manejo de aguas servidas, manejo de residuos sólidos, energía, compra de insumos, finanzas verdes y medición de los impactos. Se busca desarrollar mayor conciencia ambiental y liderazgo entre los participantes y sus familias, insertar buenas prácticas en la operación de los emprendimientos y prevenir o reducir sus impactos negativos.

En el largo plazo, se busca consolidar a la península de Osa como un destino turístico limpio, sostenible y respetuoso de su naturaleza y su biodiversidad. Las capacitaciones en temas ambientales contaron con instructores de ACOSA, del Banco Nacional de Costa Rica y de las turoperadoras nacionales.

\subsection{Arte y cultura}

El arte y la cultura juegan un papel importante en la autoestima de los habitantes, la identificación de elementos representativos de una comunidad y la definición de su identidad. A su vez contribuye a diferenciarla y convertirla en un punto de referencia cultural en el destino.

En Caminos de Osa, arte y cultura pueden ser de gran relevancia y poder transformativo, así como parte integral del desarrollo sostenible de la región. Esto se logra en las capacitaciones con los artesanos y artistas profesionales, las intervenciones en el espacio de la comunidad y con eventos culturales.

Caminos de Osa espera profundizar este componente en alianza con instituciones como el Ministerio de Cultura y el Instituto Nacional de las Mujeres, INAMU. También, bajo la sombrilla de Caminos de Liderazgo, se está teniendo acceso a recursos de JUDESUR para actividades de arte y cultura ejecutadas de la mano con el Grupo de Acción Territorial Sur Bajo, GAT, lo cual evidencia la importancia de los encadenamientos de todos los sectores incluyendo a otras plataformas y organizaciones locales.

Los logros que se esperan para la península de Osa y sus comunidades incluyen:

- Revitalización de tradiciones y costumbres de la población local.

- Uso del arte y la artesanía para estimular las habilidades y aumentar la autoestima.

- Surgimiento de nuevos emprendimientos locales en torno al arte y la cultura.

- Embellecimiento del entorno en las comunidades de Osa, con obras de arte y cultura.

- Productos culturales comunitarios que fortalezcan la identidad de Caminos de Osa. 


\section{Servicio e infraestructura turística}

\subsection{La cadena de comercialización del turismo}

Vincularse al mercado y atraer clientes es un gran desafío para emprendimientos turísticos de zonas rurales. Invertir en mercadeo internacional tiene un costo prohibitivo, requiere de muchas conexiones y es una tarea titánica para negocios pequeños.

La estrategia de Caminos de Osa ha sido hilar la cadena de comercialización con sólidos encadenamientos basados en confianza y compromiso, que incluyen emprendedores y turoperadoras locales y nacionales.

Se invitó a unirse al proyecto a 3 turoperadoras nacionales de trayectoria en la comercialización del país, comprometidas con el turismo sostenible: Horizontes Nature Tours, Travel Excellence Tour Operator y Swiss Travel. Para ellas el proyecto ha sido una oportunidad de identificar nuevos productos y servicios turísticos locales, así como diversificar sus ofertas en aventura y cultura. También apoyaron el proceso 5 turoperadoras locales del Pacífico Sur: Sierpe Azul Tours, Costa Rica Culture Tours, Osa Wild, Toucan Travel y Corcovado Info Center; motivadas por hacer partícipes a las comunidades en su abordaje empresarial. Se espera que estas alianzas faciliten a los emprendimientos de turismo rural de Caminos de Osa un mayor acceso a los nichos del mercado turístico internacional interesados en estos productos.

\subsection{Evaluación de los emprendimientos locales}

Colaboradores de las turoperadoras nacionales visitaron cada emprendimiento local de ambas generaciones de Caminos de Osa y los evaluaron exhaustivamente. Consideraron los requerimientos a cumplir para calificar como parte de sus respectivos paquetes, tales como capacidad de las instalaciones, equipamiento, decoración, rotulación, calidad, servicio al cliente (Figura 2), tarifas, planes de emergencia y pólizas de seguros. 


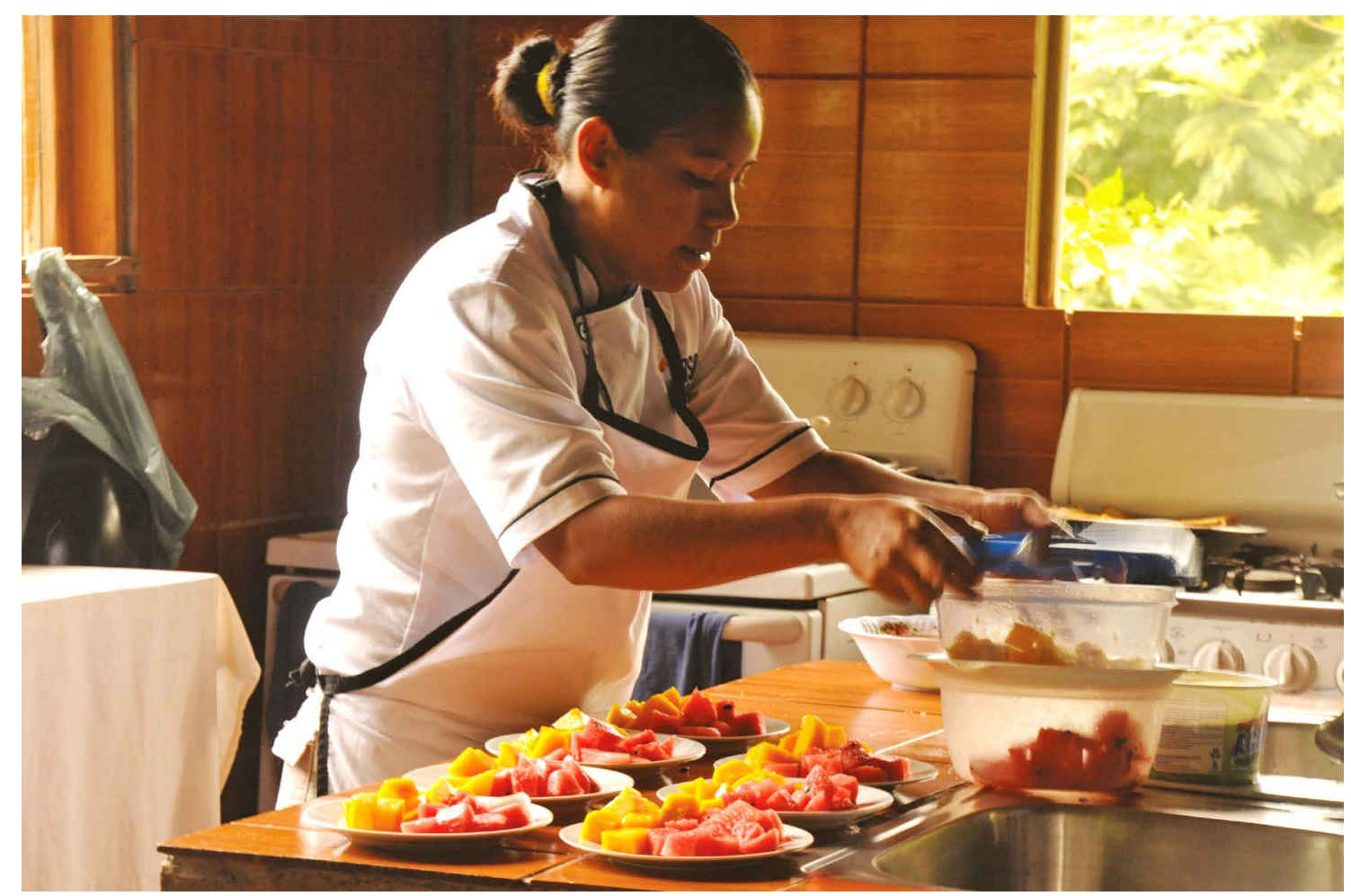

Figura 2. Caminos de Osa enfatiza en la calidad y servicio al cliente de las ofertas de emprendedores locales. Fotografía: Daniel Villafranca

Para cada emprendimiento se identificaron fortalezas, debilidades e inversiones requeridas y se elaboraron reportes individuales con las rutas críticas y un plan de implementación. Cada uno elaboró su manual de ventas y los compartió con las turoperadoras, lo que les permitió identificar productos maduros para su venta y realimentar a los que aún no estaban listos. También los emprendimientos se dieron cuenta del nivel requerido para formar parte de los paquetes.

\subsection{Las turoperadoras nacionales en la capacitación}

Las tres turoperadoras nacionales adoptaron a Caminos de Osa como una iniciativa propia, ya que sus objetivos estaban en línea con su negocio central y sus estrategias empresariales de responsabilidad social. Ellas se incorporaron con entusiasmo en la capacitación de los emprendedores, compartiendo en los talleres su conocimiento y experiencia práctica en mercadeo, operación y logística, finanzas, tarifas, costos y protocolos de emergencias. Con el acompañamiento de RBA, los colaboradores de las turoperadoras tomaron el enfoque constructivista para desarrollar actividades participativas que permitieron a los emprendedores aplicar lo aprendido en sus realidades. Para ello prepararon material didáctico y dinámicas educativas que reforzaran los conceptos.

\begin{tabular}{|c|c|c|}
\hline 228 & $\begin{array}{l}\text { Creative Commons (Reconocimiento al autor-No comercial-Compartir igual } 4.0 \text { Internacional } \\
\text { (CC BY NC SA } 4.0 \text { Internacional) }\end{array}$ & $\begin{array}{l}\text { Revista de } \\
\text { CIENCIAS AMBIENTALES } \\
\text { Tropical Journal of Environmental Sciences }\end{array}$ \\
\hline
\end{tabular}




\subsection{La mentoría}

Una vez que los emprendimientos fueron evaluados y a medida que los participantes incrementaban sus conocimientos, iban surgiendo en ellos preguntas como “Y ahora cómo llevo todo esto a la práctica? ¿Cómo realizo las mejoras que me piden las turoperadoras? ¿A quién puedo pedir consejo?”.

Para apoyarles, a lo largo de la capacitación se les ofreció mentoría por los empresarios locales y profesionales en disciplinas afines al sector turístico y empresarial, quienes acompañaron en la implementación de las medidas recomendadas (Figura 3). Algunas personas seleccionadas como mentores ya habían pasado por desafíos en la consolidación de sus propios negocios, lo que les permitió comprender las dificultades del proceso.

Mentor y aprendiz tenían el compromiso de comunicarse al menos dos veces por mes, y mínimo una ocasión en forma presencial. Cada pareja definió objetivos para avanzar en la ruta crítica sugerida en la evaluación. Aunque el mentor ofreció su acompañamiento y guía, era el emprendedor quien debía tomar las decisiones y realizar las tareas. Las visitas y comunicaciones se registraban en una bitácora con los temas de cada sesión y los avances hasta el momento. La bitácora permitió al equipo de RBA dar seguimiento al proceso y ofrecer realimentación. La mentoría es una pieza fundamental para asegurar que los emprendedores lleven a la práctica lo aprendido en los talleres y preparen sus perfiles de ventas y tarifas.

\subsection{La Asociación Caminos de Osa (ACO)}

Se concibió como una figura de gobernanza local, que liderase la gestión de Caminos de Osa como un destino turístico rural sostenible y agrupase las voces de la población en relación con asuntos de desarrollo regional. ACO busca aprovechar su capital social para tener incidencia política y asumir la operación y mantenimiento de las rutas del destino.

La formalización de la ACO y la conformación de su Junta Directiva tuvo lugar el 21 de febrero de 2015 en el salón comunal de La Palma. No se limitó a los participantes de las capacitaciones; más bien, se convocó a todas aquellas personas de las comunidades que estuviesen vinculadas a la actividad turística, en ocho sectores: 1) hoteles consolidados, 2) pequeños hoteles, 3) restaurantes, 4) turoperadores locales, 5) cámaras locales de turismo, 6) asociaciones de desarrollo integral, 7) guías, y 8) artesanos y artistas.

Desde su fundación, los integrantes de la Junta Directiva se reúnen periódicamente, con el apoyo de una Dirección Ejecutiva para analizar temas estratégicos y administrativos. Aún es necesario fortalecer la administración y las finanzas. Con el aporte de los miembros de la Junta Directiva, se definieron cuatro áreas de acción: 1) Planificación estratégica, 2) Desarrollo del destino, 3) Experiencia del visitante y 4) Marca y comercialización, cada una con objetivos y acciones prioritarias enmarcadas en un Plan Estratégico. Para los miembros de la Junta Directiva que participaron en las capacitaciones, este es un excelente entorno para poner en práctica lo aprendido en empoderamiento, comunicación, negociación, trabajo en equipo y resolución de conflictos. Se espera que la ACO llegue a ser financieramente sostenible, apoyada en la generación de recursos por la gestión de las rutas turísticas y en fondos de cooperación. 


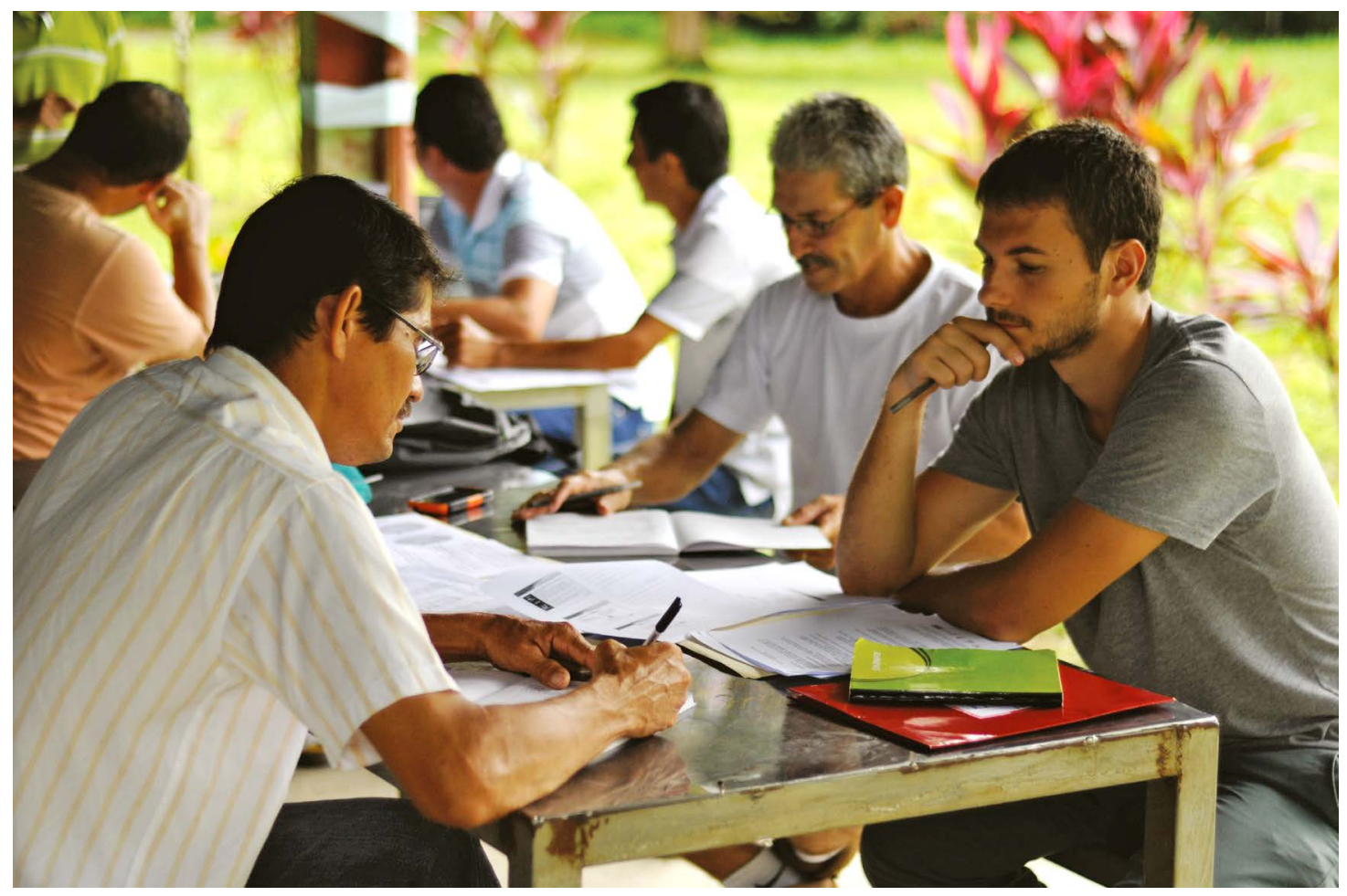

Figura 3. Sesión de mentoría con emprendedores locales de Caminos de Osa. Fotografía: Daniel Villafranca

\subsection{Certificación Caminos de Osa (CCO)}

Cada ruta conecta unos 15 emprendimientos de hospedaje, alimentación, transporte, guiado, alquiler de caballos, recorridos en kayak y otros productos y servicios, encadenados unos con otros. Es necesario generar confianza en el cliente hacia el destino y su oferta. Para ello se propuso crear la Certificación Caminos de Osa, CCO, que vela porque el cliente reciba siempre una experiencia, con estándares homogéneos de calidad, atención y seguridad. La CCO buscará beneficiar a los emprendedores, ya que les garantiza que están vinculados con otros proveedores que ofrecen un buen servicio. La CCO asegurará la calidad de la experiencia para el turista, establecerá encadenamientos entre emprendedores locales y creará beneficios para el empresario y el cliente. Para ello contará con varios instrumentos:

a) El reglamento de la CCO que define los términos relacionados con la misma.

b) La guía del auditor, herramienta que utiliza la persona que evalúa para determinar si el emprendimiento cumple con los requisitos para certificarse.

c) El equipo de implementación que permite a emprendedores identificar los cambios necesarios para cumplir con los requisitos de la certificación.

Creative Commons (Reconocimiento al autor-No comercial-Compartir igual 4.0 Internacional
(CC BY NC SA 4.0 Internacional)


Una vez completada la auditoría, la comisión de evaluación revisará los resultados y otorgará a cada emprendimiento el sello correspondiente. La evaluación se basará en cuatro atributos:

1. Espíritu eco-emprendedor: busca maximizar el impacto local de los ingresos del turismo, promoviendo encadenamientos entre productos y servicios. Se espera que cada emprendimiento certificado cuente con al menos tres vínculos con otros emprendedores de Caminos de Osa, así como cinco encadenamientos con otros proveedores productivos locales (agrícolas, comercios, talleres mecánicos).

2. Intelecto de guía: se enfocará en la generación del conocimiento del patrimonio natural y cultural, así como en su interpretación. Abarca las acciones de los emprendedores para apoyar la investigación sobre la biodiversidad. Incluye la educación del cliente con información como listas de especies o guías de campo y capacitación para el personal.

3. Corazón comunitario: el énfasis será en la contribución con la comunidad, la responsabilidad social y el trabajo en equipo. Se valorarán acciones como el ser parte activa de grupos locales organizados (asociaciones de desarrollo integral, comités locales, Asociación Caminos de Osa, otros).

4. Cuerpo biodiverso: se refiere a las acciones para la protección de la biodiversidad y del medio ambiente en general. Incluye la dedicación de tierras de las fincas a la conservación y participación en programas de pagos por servicios ambientales. También incluirá buenas prácticas como el uso de productos biodegradables, ahorro del agua, manejo de residuos, huella de carbono y otras.

Todas estas acciones permitirán crear confiabilidad con el mercado, con la comunidad y con los integrantes de los mismos emprendimientos. Se espera que la certificación beneficie al destino Caminos de Osa y fortalezca su reputación. También se confía que mejore la calidad de los servicios, aumente la eficiencia y competitividad de los emprendimientos y se apoye la comercialización de los productos al distinguirlos con un sello.

\subsection{Capacitación de guías en la filosofía de Caminos de Osa}

El guía es un elemento diferenciador del destino Caminos de Osa. Se trata de una persona con conocimiento, experiencia y sensibilidad, es un amigo de confianza que acompaña al turista en un proceso interior de cambio personal a través de su vivencia por las rutas de Osa. La capacitación de los guías de Caminos de Osa se desarrolló en el primer semestre del 2016 y se enfocó en personas que ya tienen experiencia y que cuentan con la licencia del Instituto Costarricense de Turismo (ICT). No se trata de formar nuevos guías, sino de capacitar a los que ya existen en nuestra filosofía y metodologías. Para la capacitación se contó con fondos aportados por el United States Forest and Wildlife Service, USFWS. Se preparó a los guías en aspectos emocionales, espirituales, de compromiso con la experiencia, de convivencia con la naturaleza y con otros seres humanos, así como en historia y sobre la cultura de la zona, en técnicas de guiado y en 
servicio al cliente, de manera que estén listos para acompañar al turista en el peregrinaje y en su transformación profunda.

\section{Conceptualización del destino Caminos de Osa}

\subsection{La marca Caminos de Osa}

El objetivo de posicionar a Caminos de Osa como un destino especial de naturaleza y cultura en el nivel internacional pasa por crear una marca que se asocie con la identidad de ese destino, fortaleciendo su imagen a los ojos del mercado. Para ello se contrató a Wooki Colectivo Creativo, empresa especializada en marcas, que trabajó con los emprendedores locales en varios talleres. El resultado fue el diseño de la marca Caminos de Osa. Sus productos incluyen el libro de marca y su manifiesto, los logos oficiales y propuestas para el diseño de camisas, tarjetas de presentación, videos y aplicaciones. La marca está en proceso de inscripción por parte de CRUSA.

\subsection{El desafío de diseñar las rutas turísticas}

El destino Caminos de Osa ofrece una red de tres rutas en la península de Osa, que conectan atractivos naturales y culturales, así como facilidades y servicios de emprendimientos locales. Al tiempo que realizan este peregrinaje los turistas pueden interactuar con personas de la comunidad para aprender de su cultura y de la naturaleza que les rodea.

En su diseño, se propuso desconcentrar la visitación para que esta no se enfocara solo en Corcovado y más bien se distribuyera hacia diferentes comunidades que hasta ahora solo habían sido sitios de paso. El trazado de las rutas fue uno de los mayores desafíos del proyecto. La península de Osa es un entorno muy heterogéneo por su geografía, naturaleza y clima. Hay comunidades aisladas y la infraestructura vial es deficiente en algunos lugares. Además, la situación de tenencia de la tierra es compleja, así como la administración por parte de las entidades del Estado.

Se realizaron muchas visitas al campo durante meses para corroborar la existencia y ubicación de los atractivos, definir puntos de intervención con GPS, medir las distancias y determinar la duración de las caminatas y su grado de dificultad. En ellas participaron miembros del equipo ejecutor del proyecto, integrantes de los emprendimientos locales y funcionarios de ACOSA.

\subsection{Propuesta de las rutas de Caminos de Osa}

Se diseñó el catálogo de Caminos de Osa que describe los itinerarios de cada ruta con sus actividades diarias, horarios, sitios a visitar, nivel de dificultad, comidas y características del hospedaje. Cada ruta encadena servicios locales de hospedaje, alimentación, guiado, atracciones temáticas, transporte, y otros. Los negocios con ofertas similares, como hospedaje, se rotan para asegurar que todos se beneficien. En la medida de lo posible, se prevé que los caminos ingresen al Parque Nacional Corcovado en su recorrido, de manera que este sea una parte integral, aunque no central, de la oferta. 
A continuación, se ofrece una breve descripción de las rutas:

- Camino de la selva: "El verdadero color del oro es verde". Experiencia de cuatro días, con grados de dificultad de 4-7. Encadena 20 emprendimientos e incluye caminata, baño en río, cabalgata, kayak y comunidad indígena.

- Camino del agua: "El susurro del viento y el rugir de las olas nos muestran el camino". Es un recorrido de cuatro días, con grados de dificultad 5-6. Incluye bote por manglares, caminata, baño en río, cabalgata y esnórquel.

- Camino del oro: “Un tour es mucho más que un tour, es una forma de vida”. Desarrolla el tema del oro en la península de Osa y la vida de los oreros. Es una experiencia de seis días, con grados de dificultad 6-8. Incluye museo precolombino, bote por manglar, caminata, sitio orero, comunidad indígena y cabalgata.

- Osa elemental: "Usted no puede conocer un lugar profundamente sin conocer a su gente”. Esta opción de cinco días permite visitar diferentes puntos de interés desplazándose en medios de transporte. Tiene un grado de dificultad 2-7 e incluye comunidad indígena, caminatas, baño en río, kayak y cabalgata.

- Itinerarios personalizados: estos se ofrecen a turistas con poca disponibilidad de tiempo, familias, grupos de estudiantes o huéspedes de hoteles de la zona.

\section{Resultados y lecciones aprendidas}

- El trabajo mancomunado de los cuatro socios de la alianza funcionó gracias a su compromiso de conocerse mejor, comunicarse fluidamente y con transparencia, cumplir unos con otros y rendir cuentas de su trabajo. Ellos lograron construir una visión compartida y un fin común al integrar sus capacidades técnicas, el conocimiento, la red de relaciones y el financiamiento en un mismo proceso de desarrollo local sostenible.

- Otro factor de éxito fue la amplia red de apoyo que se logró construir con las empresas privadas, instituciones públicas, bancos, universidades y otros actores, todos apoyando a Caminos de Osa desde sus respectivos frentes.

- Para tener acceso al mercado se completó la cadena de comercialización. Esto es necesario para lograr que los negocios se mantengan en el tiempo y prosperen, una vez que los proyectos de cooperación hayan finalizado. Integrar a las turoperadoras nacionales en la comercialización de la oferta de Caminos de Osa y en la capacitación de los participantes agregó valor al proyecto e incrementó sus posibilidades de éxito.

- Un programa de capacitación no puede limitarse a temas de turismo y administración o a planes de negocios. Esto es insuficiente para lograr resultados a largo plazo de transformación profunda en una comunidad. Incluir temas de fortalecimiento de la autoestima, empoderamiento e identidad cultural fue fundamental para sustentar el crecimiento individual de los participantes. El cambio en ellos fue notorio, comenzaron a percibirse a sí mismos como empresarios exitosos y actores responsables de su desarrollo, más que víctimas de sus circunstancias. De hecho, los partícipes comenzaron a actuar y a expresarse de formas tal que reflejaban esa convicción. 
- El trazado e implementación de rutas de aventura y cultura es un trabajo interdisciplinario, que requiere de muchas visitas al campo, así como de muchos saberes diferentes, por lo que un factor que contribuye al éxito es incorporar a personas locales que conocen la zona, así como gente con formación en turismo, geografía, historia natural y cultural, interpretación, arquitectura, seguridad, mercadeo, y otros.

\section{Referencias}

Dirzo, R., Broadbent, E., Almeyda, A., Morales, L., Almeyda, S., Quispe, C. (2014). Ecosistemas terrestres de la región de Osa y Golfito, Costa Rica. Stanford Woods Institute for the Environment, Stanford University. Informe final.

Gaffikin, L. (2013). Evaluación de la salud: Su historia, estado y determinantes de la salud en el área focal de Osa/Golfito INOGO en Costa Rica. San José, Costa Rica: INOGO, Stanford Woods Institute for the Environment.

Hunt, C., Menke, C. y Durham, W. (2013). Sustainable Development Centered on Human Well-Being in Osa and Golfito, Costa Rica: A Social Diagnostic Analysis. Stanford, California: Stanford Woods Institute for the Environment.

Menke, C. y M. Carnoy. (2013). Educación para un futuro sostenible: Análisis del sistema educativo en Osa y Golfito. San José, Costa Rica: INOGO, Stanford Woods Institute for the Environment.

Román, M. y Angulo, J. (2013). Panorama socioeconómico de los cantones de Osa y Golfito: tendencias y desafíos para el desarrollo sostenible. San José, Costa Rica: INOGO, Stanford Woods Institute for the Environment. 